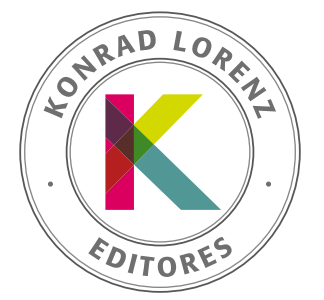

\title{
Sexting en universitarios: relación con edad, sexo y autoestima
}

\author{
Helena Chacón-López ${ }^{a}$, María Jesús Caurcel-Cara ${ }^{\mathrm{a}, *}$ y Juan Francisco Romero-Barriga ${ }^{\mathrm{b}}$
}

\author{
a Departamento de Psicología Evolutiva y de la Educación, Universidad de Granada, Granada, España \\ b Departamento de Didáctica y Organización Escolar, Universidad de Granada, Granada, España
}

Recibido el 15 de septiembre de 2018; aceptado el 1 de marzo de 2019

\section{PALABRAS CLAVE} estudiantes, sexting, universidad autoestima, sexo,

\begin{abstract}
Resumen El sexting es un fenómeno social globalizado que aumenta con la edad en adolescentes, aunque hay pocas investigaciones centradas en conocer su práctica en universitarios, motivo del presente estudio, en el que participaron 899 jóvenes estudiantes de la Universidad de Granada (España) con edades entre 18 y 24 años. Los objetivos eran conocer (mediante análisis de frecuencias y correlaciones) la prevalencia del sexting entre hombres y mujeres, analizar su relación con la edad, el sexo y la autoestima; y determinar la prevalencia de la práctica del sexting por edad. Se utilizaron dos instrumentos, la Escala de Conductas sobre Sexting y la Escala de Autoestima de Rosenberg. La relevancia del estudio radica en que constata que tanto chicos como chicas realizan sexting, más los chicos, y que la práctica se incrementa hasta los 21 años, descendiendo ligeramente a los 24 . No se encontró relación con autoestima. Como conclusión se recomienda prudencia (puesto que la mayoría ha realizado sexting alguna vez) al ser una práctica no exenta de riesgo de la que pueden derivarse consecuencias personales y profesionales negativas, por la amplia difusión y permanencia que puede tener este material en la red. ๑ 2019 Fundación Universitaria Konrad Lorenz. Este es un artículo Open Access bajo la licencia CC BY-NC-ND (http://creativecommons.org/licenses/bync-nd/4.0/).
\end{abstract}

\section{Sexting among university students: Relation with age, sex and self-esteem}

\section{KEYWORDS}

students, sexting, self-esteem, sex, university
Abstract Sexting is a globalized social phenomenon that increases with age in adolescents, although there are few researches focused in its practice in university students, goal of the present study, which involved 899 students of the University of Granada (Spain) with ages between 18 and 24 years. The objectives were to know (through analysis of frequencies and correlations) the prevalence of sexting between men and women, to analyze the relation with age, sex and self-esteem; and to determine the prevalence of the practice of sexting by age. Two instruments were used, the Sexting Behaviors Scale and the Rosenberg Self-Esteem Scale. The relevance of the study lies in the fact that confirm that both

* Autor para correspondencia.

Correo electrónico: caurcel@ugr.es.

http://dx.doi.org/10.14349/sumapsi.2019.v26.n1.1

0120-0534/@ 2019 Fundación Universitaria Konrad Lorenz. Este es un artículo Open Access bajo la licencia CC BY-NC-ND (http://creativecommons.org/licenses/by-nc-nd/4.0/). 
men and women practice sexting, more men, and that the practice increases until the 21 years, falling slightly at 24 . No relation was found with self-esteem. The study concludes that since most of the students have done sexting, precaution is recommended, because of the risk of the negative personal and professional consequences that may result, for the wide dissemination and permanence that can have this information in the network. (c) 2019 Fundación Universitaria Konrad Lorenz. This is an open access article under the CC BYNC-ND license (http://creativecommons.org/licenses/bync-nd/4.0/).

La universalización del uso de dispositivos digitales -principalmente relacionados con la tecnología móvil- idóneos para la creación y difusión de contenido multimedia, está cambiando los hábitos y las formas de comunicación interpersonal, las relaciones íntimas, e incluso el comportamiento sexual (Ballester, Gil, Gómez \& Gil, 2010; Gámez-Guadix, Santisteban \& Resett, 2017), especialmente entre adolescentes y jóvenes (Deb-Levine, 2013). El uso indiscriminado de esta tecnología ha propiciado, entre otros fenómenos, la aparición del sexting, un neologismo que une "sex" (sexo) y "texting" (envío de mensajes de texto a través de dispositivos móviles) (Agustina \& Gómez-Durán, 2012; Mitchell, Finkelhor, Jones \& Wolak, 2012; Morelli, Bianchi, Baiocco, Pezzuti \& Chirumbolo, 2016). Originalmente, el término se refería al envío de textos (SMS), pero hoy se aplica a la difusión o publicación de contenido provocativo o sexual, creado por el remitente y utilizando para ello el teléfono móvil o cualquier otro dispositivo (Inteco, 2011). El formato puede ser de contenido mensaje de texto, fotos y videos; y el tipo de dispositivos utilizados teléfonos inteligentes y otros terminales con acceso a Internet (Chalfen, 2009).

El sexting se extiende más allá del envío de textos sexualmente sugerentes, incluyendo una amplia gama de comportamientos, prácticas y motivaciones (Lee \& Crofts, 2015). Algunas razones por las que los jóvenes, particularmente las chicas, envían imágenes sobre sí mismos (generalmente a varones jóvenes), son la coerción o presión individual, del grupo de iguales, o sociocultural (Durham, 2008; Englander, 2012; Gill, 2012; Ringrose, Harvey, Gill \& Livingstone, 2013). Ello privilegia exclusivamente los aspectos negativos del sexting, olvidando que podría integrarse en los sistemas de cortejo, relaciones de pareja y amistad (Albury, Funnell \& Noonan, 2010), siendo una opción libre para expresarse sexualmente (Hasinoff, 2014).

Hay dificultades para diferenciar entre sexting consensuado, motivado por el placer o el deseo (Lee \& Crofts, 2015), que según Hasinoff (2014), nunca debe ser criminalizado; y el coercitivo, o uso indebido en las redes sociales para acosar sexualmente a otros, provocando que puedan convertirse en víctimas de depredadores en línea; al poseer imágenes sexualmente explícitas difundidas sin su consentimiento (García-Gómez, 2019).

El sexting es un fenómeno social globalizado que ha recibido notable atención en los últimos años por los medios de comunicación e investigadores (Agustina \& Gómez-Durán, 2012). Sin embargo, la investigación mayoritaria proviene de Estados Unidos (con una perspectiva nacional) y posee limitaciones relacionadas con la definición utilizada, la representatividad de la muestra, e instrumentos de medida (Döring, 2014; Klettke, Hallford \& Mellor, 2014).

En universitarios norteamericanos -entre 18-24 años(Benotsch, Snipes, Martin \& Bull, 2013; Dir, Cokunpinar, Steiner \& Cyders, 2013; Perkins, Becker, Tehee \& Mackelprang,
2014; Reyns, Henson \& Fisher 2014), las cifras de participación varían entre $12.4 \%$ a $62 \%$ para el envío y recepción de mensajes; $5.1 \%$ a $67.4 \%$ solo envío; y $10.3 \%$ a $80.3 \%$ solo recepción; llegando a ser omnipresente en sus relaciones (O’Neal, Cummings, Hardy \& Ott, 2013). Otros estudios han confirmado que es una práctica común en las relaciones románticas estables de los adultos jóvenes (Drouin \& Landgraff, 2012; Drouin, Vogel, Surbey \& Stills, 2013), aunque necesitaban conocer a la persona para practicarlo (Delevi \& Weisskirch, 2013).

En España son muy escasos los estudios sobre participación de adultos, si bien se han hallado tasas de prevalencia en torno al 66.8\% (Gámez-Guadix, Almendros, Borrajo \& Calvete, 2015). En estudiantes universitarios, el $1.5 \%$ de entre 18 a 22 años y el $21.7 \%$ de 23 a 29 años, admiten realizar sexting activo (envío de mensajes protagonizados por ellos mismos), el 2.1\% de 18-22 años y el 20\% de 23-29 años participan en el pasivo (envío de mensajes protagonizados por otras personas) (Agustina \& Gómez-Durán, 2016).

El sexting está ganando popularidad entre los adultos, los adolescentes y, particularmente, las adolescentes (García-Gómez, 2019). Si bien, era más frecuente entre los adultos que en los adolescentes, estos se involucraban más con la edad (Döring, 2014; Klettke et al., 2014), hallando un aumento de prevalencia del 3\% a los 12 años al 32\% a los 18 (Dake, Price, Maziarz \& Ward, 2012); incremento igualmente detectado por Capafóns (2014) y por Quesada, Fernández-González y Calvete (2018). Es más, parece ser una forma relativamente común de interacción sexual entre adolescentes, pues aproximadamente el $15 \%$ de 12 a 17 años admite haber enviado contenido sexual online; porcentaje que se amplía hasta el 36\% a los 17 años (Gámez-Guadix et al., 2017). Este incremento de participación asociado a la edad parece relacionarse con el aumento del establecimiento de relaciones íntimas, el mayor acceso a la tecnología necesaria para practicarlo y la proliferación de redes sociales.

Atendiendo a la perspectiva de género, la mayoría de los estudios (posfeministas) se centran en cómo las adolescentes y mujeres jóvenes representan y negocian su identidad de género y sexualidad en el contexto del sexting, dejando casi de lado cómo lo hacen los hombres. Algunos ofrecen una explicación simplista (Dobson, 2014, 2015; García-Gómez, 2018, 2019; Hasinoff, 2012, 2014, 2015) al describir a las primeras como víctimas de la sexualización, que reproducen las normas de género dominantes y a las que se pide (al contrario que a los hombres) que aborden los efectos negativos de la sexualización temprana (Livingstone, 2008). Cuando, en realidad, los análisis sobre las creencias y las motivaciones detrás del sexting consensuado, realizados por García-Gómez $(2017,2018)$, revelan que las jóvenes heterosexuales británicas lo entienden como un medio para vivir plenamente su vida sexual y relacionarse. 
Distintos estudios (Benotsch et al., 2013; Perkins et al., 2014; Quesada et al., 2018) manifiestan que tanto hombres como mujeres se involucran de manera similar en conductas de sexting. Aunque se ha hallado con más frecuencia en hombres (Capafóns, 2014; Gámez-Guadix, Borrajo \& Almendros, 2016; Gordon-Messer, Bauermeister, Grodzinski \& Zimmerman, 2013), detectando además diferencias en función de la orientación sexual (Morelli et al., 2016); y otros, en cambio (Reyns et al., 2014), advirtieron de que las mujeres eran más propensas a enviar mensajes de contenido sexual y los hombres a recibirlos.

Son escasos los estudios concernientes a la relación entre autoestima y sexting; no obstante, se ha señalado la autoestima como un factor de protección en la vivencia de una sexualidad sana (Cataño, Restrepo, Portilla \& Ramírez, 2008) y ante el sexting y los fenómenos conexos (Alonso, 2017). Hudson (2011) no halló correlaciones significativas entre autoestima y conductas o actitudes relacionadas con el sexting; ni con riesgo sexual, depresión o ansiedad (Gordon-Messer et al., 2013). Mientras baja autoestima y altos niveles de neuroticismo predecían mayor implicación en sexting (Delevi \& Weisskirch, 2013); siendo los adolescentes con alta autoestima los que menos sextean (Ybarra \& Mitchell, 2014), y aquellos con mayor autocontrol (Marcum, Higginns \& Ricketts, 2014).

En España, apenas existen estudios que aborden la práctica del sexting en universitarios, carencia que, junto con otras circunstancias preocupantes como que pueda convertirse en una conducta de riesgo al dejar huella permanente en la red (aun siendo una práctica consentida), y que su hábito parezca aumentar con la edad, ha motivado el presente estudio cuyos objetivos son: (1) conocer la prevalencia del sexting tanto en hombres como en mujeres estudiantes de la Universidad de Granada; (2) determinar la relación entre sexting, edad, sexo y autoestima; (3) analizar la prevalencia de esta práctica de acuerdo con la edad.

Según estudios previos, se espera constatar esta práctica tanto en chicos como en chicas, aunque previsiblemente serán los primeros quienes la realicen con más frecuencia. Asimismo, esperamos detectar relaciones con la edad y la autoestima y un incremento del sexting a medida que aumenta la edad.

\section{Método}

\section{Participantes}

Participaron 899 jóvenes: 397 hombres (44.16\%) y 502 mujeres (55.86\%), estudiantes de la Universidad de Granada (España), con edades comprendidas entre los 18 y 24 años $(M=20.84 ; D T=1.897$; hombres: $M=20.930 ; D T=1.877$; mujeres: $M=20.780 ; D T=1.913$ ); no existiendo diferencias significativas entre ambas muestras $(t=1.178$; $p=.239)$. Se seleccionaron entre una población inicial de participantes mediante muestreo aleatorio de casos (usando el programa estadístico SPSS), excluyendo aquellos que no habían completado alguna de las escalas en su totalidad. La tabla 1 recoge las restantes características sociodemográficas.

\section{Instrumentos}

Se utilizó la Escala de Conductas sobre Sexting (ECS; Dir, Cyders \& Coskunpinar, 2013), traducida y validada en pobla-
Tabla 1 Características sociodemográficas de la muestra

\begin{tabular}{lcc}
\hline & $\mathbf{N}^{\circ}$ & $\%$ \\
\hline Orientación sexual & & \\
Heterosexual & 846 & 94.10 \\
Homosexual & 25 & 2.78 \\
Bisexual & 26 & 2.89 \\
Transexual & 0 & 0.00 \\
No contesta & 2 & 0.22 \\
\hline Estado civil & & \\
\hline Soltero & 535 & 59.51 \\
Casado & 14 & 1.56 \\
Novio & 275 & 30.59 \\
Vivir en pareja & 13 & 1.67 \\
Empezando a salir con alguien & 56 & 6.23 \\
No contesta & 4 & 0.44 \\
\hline Total & 899 & 100.00 \\
\hline
\end{tabular}

ción española (Chacón-López, Romero-Barriga, Aragón-Carretero \& Caurcel-Cara, 2016); instrumento con consistencia interna adecuada (alfa de Cronbach $=.922$ ). Mide frecuencia y prevalencia de conductas relacionadas con recepción y envío de mensajes de texto o imágenes con contenido provocativo o sexual, a través del teléfono móvil o las redes sociales. Consta de 29 ítems, agrupados en 3 factores: F1) participación real en sexting, 16 ítems; F2) disposición activa hacia el sexting, 9 ítems; y F3) exposición emocional ante el sexting, 4 ítems. La opción de respuestas es de formato tipo Likert de cinco puntos, oscilando entre 0 ("nunca"/"nada cierto") hasta 4 ("frecuentemente"/ "totalmente cierto").

Se usó la Escala de Autoestima de Rosenberg (EAR, 1965), adaptada para población española (Vázquez-Morejón, Jiménez García-Bóveda \& Vázquez-Morejón Jiménez, 2004), que proporciona un índice global de sentimientos de respeto y aceptación de sí mismo. Su consistencia interna (usando alfa de Cronbach) es de 0.87. Consta de diez ítems de respuesta múltiple con cuatro opciones de respuesta (A = muy de acuerdo; $\mathrm{B}$ = de acuerdo; $\mathrm{C}$ = en desacuerdo; $\mathrm{D}=$ totalmente en desacuerdo); cinco de los ítems están enunciados negativamente y cinco positivamente. La puntuación global oscila entre 10 y 40, resultado de sumar todos los ítems y teniendo en cuenta que del 1 al 5 (correspondientes a las opciones de respuesta de $A$ hasta $D$ ) puntúan de 4 a 1 , y los ítems del 6 al 10, puntúan de 1 a 4 . Una puntuación entre 30 y 40 mostrará autoestima elevada; de 26 a 29, autoestima media; y menos de 25, autoestima baja.

Los valores psicométricos adecuados de ambas escalas y el estar validadas en población española determinaron la decisión de su uso.

\section{Procedimiento}

Los participantes eran estudiantes de la Universidad de Granada a los que los investigadores informaron del obje- 
tivo del estudio y ofrecieron la posibilidad de colaborar voluntariamente, respetando durante todo el proceso las normas éticas internacionales (APA, 2017). Se incidió en la importancia de la sinceridad en sus respuestas, dado su carácter anónimo y confidencial. La cumplimentación de los instrumentos se realizó online, individualmente, mediante un enlace web creado por los investigadores y enviado por correo electrónico, en el que se informaba del protocolo a seguir para rellenarlo. Los datos se recogieron a lo largo de un cuatrimestre utilizando una versión electrónica de las escalas mediante la aplicación LimeSurvey (Versión 2.00+), para el posterior procesamiento.

\section{Análisis de datos}

Para analizar la prevalencia del sexting tanto en hombres como en mujeres, se realizó un análisis porcentual en cada uno de los ítems de la escala, de acuerdo con la frecuencia producida; aunque para evitar la saturación de la tabla resultante se agruparon las cinco opciones (nunca, rara vez, ocasionalmente, a menudo, frecuentemente) en tres (nunca, rara vez-ocasionalmente, a menudo-frecuentemente). Se completó el análisis de diferencias entre sexos con el cálculo de la prueba $\mathrm{Chi}^{2}$. Seguidamente se llevó a cabo un análisis de correlación de Pearson para conocer la relación entre los tres factores (en los que se agrupan los ítems) del sexting, autoestima, edad y sexo. Finalmente, se calculó la prevalencia de cada factor de acuerdo con la edad de los participantes. El análisis de los datos se realizó usando el paquete estadístico SPSS versión 21.0. para Windows.

\section{Resultados}

En la tabla 2 se presenta la prevalencia del sexting en cada uno de los ítems tanto en hombres como en mujeres, de acuerdo con la frecuencia producida, y los $p$-valores resultantes de la prueba $\mathrm{Chi}^{2}$ en los ítems con diferencias significativas. Se observa predominio de participación mayor en hombres (41.57\%) que en mujeres (34.47\%), apreciando diferencias significativas en los siguientes ítems: respuesta mensajes de texto o con imágenes provocativos/insinuantes recibido en el móvil, recepción imágenes o mensajes provocativos/insinuantes por Internet; hago sexting con mi novio/a, cuando estoy bebiendo alcohol, fumando marihuana o consumiendo otras drogas; estoy de marcha con amigos/ as, aburrida/o, de buen humor, solo/a, aislada/o, porque quiero tener relaciones sexuales, empezar a salir con alguien, hablar con alguien, o porque el sexting hace que tenga más probabilidad de tener sexo o salir con alguien.

Se constatan puntuaciones más altas en los chicos en casi todas las opciones y en cada uno de los ítems: asocian más la práctica con la ingesta de alcohol (H: 20.65\%; M: 12.36\%), consumo de marihuana $u$ otras drogas $(\mathrm{H}: 10.9 \%$; $M$ : $3.19 \%)$, al salir de fiesta con amigos ( $\mathrm{H}: 16.88 \%$; $\mathrm{M}: 8.57 \%)$, cuando están aburridos $(\mathrm{H}: 27.95 \%$; $M$ : 19.95\%), de buen humor $(\mathrm{H}$ : 39.8\%; $M$ : 31.07\%), solos/as ( $\mathrm{H}: 46.4 \%$; $M: 32.08 \%)$, aisladas/ os $(\mathrm{H}: 21.7 \%$; $M: 11.6 \%)$, cuando quieren tener relaciones sexuales ( $\mathrm{H}: 43.58 \%$; $M$ : 30.28\%), cuando quieren empezar a salir con alguien ( $\mathrm{H}: 21.41 \%$; $M$ : 9.36\%), hablar con alguien $(H: 20.65 \%$; $M: 8.36 \%)$, porque creen que así tendrán más probabilidad de tener sexo o de salir $(\mathrm{H}: 52.9 \%$; $M$ : $38.84 \%)$.

La mayoría de los participantes dicen haber recibido alguna vez contenido provocativo a través del móvil, en mensajes de texto (H: 79.09\%; $M$ : 75.1\%), o en mensajes con imágenes provocativas o insinuantes $(\mathrm{H}: 74.06 \%$; $\mathrm{M}: 69.72 \%)$. El porcentaje más alto practican el sexting con 1 o 2 personas (42.82\% hombres y $47.41 \%$ mujeres), un $16.89 \%$ de hombres y $8.96 \%$ de mujeres lo hacen con 3 a 5 personas; un $2.52 \%$ de hombres y $1.20 \%$ de mujeres con 6 a 10 personas y un $4.53 \%$ de hombres y $3.39 \%$ de mujeres con más de 10 . Siendo mayor el porcentaje de mujeres (20.51\%) que de hombres (16.88\%) que afirman practicarlo con su novio "ocasionalmente"/“a menudo". La diferencia más marcada entre sexos se refiere a que los hombres reciben más mensajes provocativos, tanto en el móvil como en cualquier otro medio por Internet. Asimismo, tienen mayor predisposición a responderlos a través del móvil, tanto de texto $(\mathrm{H}: 61.71 \%$; M: 53.59\%) como de imágenes ( $\mathrm{H}: 57.32 \%$; $M: 46.62 \%)$.

Tabla 2 Prevalencia del sexting en hombres y mujeres en cada uno de los ítems, de acuerdo con la frecuencia y los p-valores

\begin{tabular}{|c|c|c|c|c|c|c|c|}
\hline \multirow{3}{*}{ Ítem } & \multirow{2}{*}{\multicolumn{2}{|c|}{ Nunca }} & \multirow{2}{*}{\multicolumn{2}{|c|}{$\begin{array}{c}\text { Rara vez } \\
\text { Ocasionalmente }\end{array}$}} & \multirow{2}{*}{\multicolumn{2}{|c|}{$\begin{array}{c}\text { A menudo } \\
\text { Frecuentemente }\end{array}$}} & \multirow{3}{*}{$p$} \\
\hline & & & & & & & \\
\hline & $\% \mathrm{H}$ & $\% M$ & $\% \mathrm{H}$ & $\% M$ & $\% \mathrm{H}$ & $\% M$ & \\
\hline 1. Recepción mensajes en móvil & 20.91 & 24.90 & 66.75 & 65.14 & 12.34 & 9.96 & \\
\hline 2. Respuesta a mensajes & 38.29 & 46.41 & 52.39 & 47.21 & 9.32 & 6.38 & 0.015 \\
\hline 3. Recepción de imágenes en móvil & 25.94 & 30.28 & 67.75 & 63.55 & 6.30 & 6.17 & \\
\hline 4. Respuesta a imágenes & 42.57 & 53.39 & 51.63 & 42.03 & 5.79 & 4.59 & 0.001 \\
\hline 5. Envío de SMS mediante móvil & 47.88 & 48.61 & 46.10 & 46.41 & 6.04 & 4.98 & \\
\hline 6. Envío de imágenes mediante móvil & 49.87 & 57.77 & 46.34 & 39.44 & 3.77 & 2.79 & \\
\hline $\begin{array}{l}\text { 7. Recepción de imágenes/mensajes mediante redes socia- } \\
\text { les/e-mail }\end{array}$ & 42.82 & 45.02 & 50.63 & 50.40 & 6.55 & 4.58 & 0.018 \\
\hline 8. Envío de imágenes/mensajes mediante redes sociales/e-mail & 71.54 & 77.49 & 27.96 & 20.91 & 0.50 & 1.60 & \\
\hline 9. Publicación de imágenes en Facebook/otras & 86.40 & 87.65 & 12.85 & 11.16 & 0.76 & 1.20 & \\
\hline
\end{tabular}


Tabla 2 Prevalencia del sexting en hombres y mujeres en cada uno de los ítems, de acuerdo con la frecuencia y los p-valores (continuación)

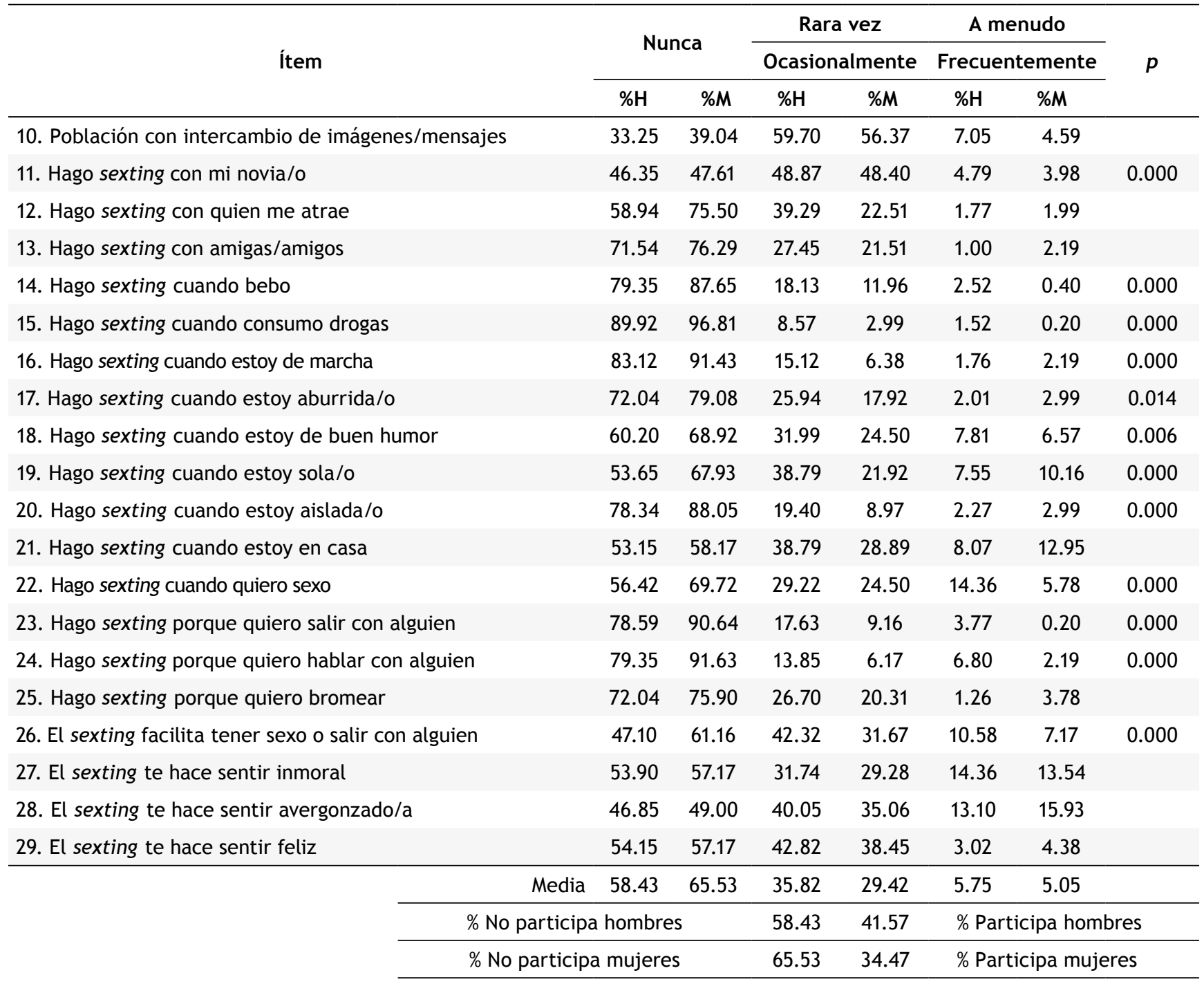

Los resultados del análisis de correlaciones detectaron relaciones significativas y positivas (véase tabla 3 ), aunque bajas, entre la edad y los tres factores del sexting (F1 participación real, F2 - disposición activa y F3 - exposición emocional); significativas (también bajas) y negativas entre el sexo y estos; no obteniendo correlación entre autoestima y el resto de variables.

Los resultados de la prevalencia de cada factor del sexting por edad, pueden observarse en la tabla 4, la cual muestra el porcentaje de jóvenes que lo realiza. Se constata un incremento progresivo desde los 18 a los 20 años y, a partir de ahí, una tendencia decreciente, cifrándose el índice de participación a los 24 años en $11.23 \%$, ligeramente inferior al de los 18 años. Se aprecian igualmente diferencias significativas en los dos primeros factores, aunque no en el tercero $(\mathrm{F} 3)$.

\section{Discusión}

Este estudio se marcó como primer objetivo determinar la prevalencia del sexting en una muestra de jóvenes uni- versitarios españoles con edades comprendidas entre 18 y 24 años.

Los datos ponen de relieve que cuatro de cada diez entrevistados reconoció haberse visto involucrado alguna vez en el intercambio de contenido provocativo a través del móvil, ya sea en mensajes de texto o en mensajes con imágenes provocativas o insinuantes; si bien, la mayor parte de ellos mostraron conformidad con una baja participación activa, encontrando que esta se produce en mayor medida en hombres que en mujeres. Estos resultados muestran la extensión del sexting entre jóvenes universitarios (acorde con la hipótesis de partida) y son similares a los encontrados en investigaciones recientes tanto en el sentido de ser una práctica frecuente entre adultos, como en la tendencia general en el grado de participación por parte de ambos sexos (Morelli et al., 2016).

Aunque aparecen algunos casos donde el intercambio de este tipo de contenido se produce con varias personas, la mayoría afirma interactuar con una o dos personas, sugiriendo que esta práctica pudiera estar ajustada al ámbito afectivo entre parejas. De hecho, más de la mitad de 
Tabla 3 Análisis de correlación de Pearson entre F1, F2 y F3, autoestima, edad y sexo

\begin{tabular}{|c|c|c|c|c|c|c|}
\hline & & Sexo & $\mathrm{F} 1$ & $\mathrm{~F} 2$ & F3 & Autoestima \\
\hline \multirow{2}{*}{ Edad } & Correlación & $-.039^{* *}$ & $.101^{* *}$ & $.136^{* *}$ & $.131^{* *}$ & .014 \\
\hline & Sig. (bilateral) & $.228^{* * *}$ & $.006^{* *}$ & $.000^{\star *}$ & $.000^{* *}$ & .840 \\
\hline \multirow{2}{*}{ Sexo } & Correlación & $* *$ & $-.160^{* *}$ & $-.081^{* *}$ & $-.090^{* *}$ & .004 \\
\hline & Sig. (bilateral) & & $.000^{\star *}$ & $.013^{* *}$ & $.008^{* *}$ & .904 \\
\hline \multirow{2}{*}{$\mathrm{F} 1$} & Correlación & & $*$ & $.641^{* *}$ & $.281^{* *}$ & -.016 \\
\hline & Sig. (bilateral) & $* *$ & & $.000^{\star *}$ & $.000^{* *}$ & .538 \\
\hline \multirow{2}{*}{$\mathrm{F} 2$} & Correlación & & & $*$ & $.243^{* *}$ & -.003 \\
\hline & Sig. (bilateral) & $* *$ & $* *$ & & $.000^{* *}$ & .839 \\
\hline \multirow{2}{*}{ F3 } & Correlación & & & & $* *$ & .014 \\
\hline & Sig. (bilateral) & $* *$ & $* *$ & $* *$ & & .729 \\
\hline
\end{tabular}

$N=899$

**. La correlación es significativa al nivel 0.01 (bilateral).

*. La correlación es significante al nivel 0.05 (bilateral).

Tabla 4 Prevalencia de cada factor del sexting de acuerdo con la edad

\begin{tabular}{|c|c|c|c|c|c|c|c|c|c|}
\hline Edad & 18 & 19 & 20 & 21 & 22 & 23 & 24 & Totales & $x^{2}$ \\
\hline$\%$ & 12.90 & 15.91 & 17.46 & 16.46 & 13.46 & 12.57 & 11.24 & 100.00 & \\
\hline $\mathrm{F} 1$ & 2.79 & 4.43 & 5.40 & 3.90 & 4.29 & 4.12 & 3.60 & 28.53 & $16.330^{* *}$ \\
\hline $\mathrm{F} 2$ & 4.86 & 7.14 & 10.28 & 7.61 & 6.54 & 7.39 & 6.01 & 49.83 & $24.213^{* * *}$ \\
\hline F3 & 6.40 & 6.73 & 6.62 & 6.37 & 7.23 & 6.56 & 6.45 & 46.36 & 2.881 \\
\hline
\end{tabular}

Nota: ${ }^{* *} \mathrm{p}<0.01,{ }^{* * *} \mathrm{p}<0.001$

mujeres y hombres afirmaron practicarlo con su novio/a, entre ocasionalmente o a menudo. Los estudios que han indagado en las motivaciones que llevan a los jóvenes a practicar sexting han constatado que gran parte del intercambio se produce en un contexto romántico con un componente lúdico (Crofts \& Lee, 2015); incluso, algunos autores afirman que cuando este intercambio es consentido por ambos miembros aparecen niveles más altos de satisfacción en la relación (Stasko, 2018). En cualquier caso, el hecho de que el sexting se lleve a cabo en situaciones no coercitivas o plenamente consentidas, no evita los riesgos inherentes que se derivan de su práctica dada la fragilidad de las relaciones humanas.

En cuanto a las diferencias encontradas entre hombres y mujeres, de forma general, las más significativas están relacionadas con mayor participación por los primeros, ya sea en la recepción de mensajes provocativos como en la predisposición a responderlos, tanto de texto como de imágenes. Más específicamente, se observan algunas diferencias significativas relacionadas con las situaciones que propician la aparición de sexting, así entre las personas que declaran practicarlo, buena parte de ellas, y en mayor medida los hombres, afirman que suelen hacerlo estando solas o en casa; aunque aquí no parece haber diferencias. También se detecta una preferencia de sexting con la búsqueda de relaciones sexuales, cuestión reafirmada con la opinión expresada por cinco de cada diez hombres y cuatro de cada diez mujeres en la opción que enuncia que hace más probable tener sexo o salir con alguien. Otra situación que provoca sexting, aunque en menor medida, es estar de buen humor, con diferencias a favor de los hombres. El resto de situaciones parecen contar con menos aprobación (aproximadamente tres de cada diez personas); no aparecen diferencias de sexo en los casos de hacerlo con amigas o amigos y bromear con gente; se aprecian diferencias acusadas por los hombres en situaciones como hacer sexting buscando hablar con alguien, estando de marcha con amigos, por estar aislada/o, y bebiendo alcohol. Muy pocos lo asociaron con el consumo de marihuana u otras drogas, aunque con diferencias bastante significativas a favor de los hombres.

No se dan diferencias en la expresión de emociones percibidas con la práctica de sexting, detectando que tanto la mitad de hombres como de mujeres declaran que les hace sentirse avergonzados; de igual modo, a cuatro de diez personas aproximadamente les hace sentirse inmoral y en la misma proporción les hace sentirse feliz.

El segundo objetivo buscaba determinar la relación del sexting con la edad, el sexo y la autoestima. Los resultados 
confirman correlación con las dos primeras variables, pero no con la autoestima, por lo que no parece ser relevante en la práctica. Datos similares a los encontrados por Hudson (2011) y Gordon-Messer et al. (2013).

Acerca de la prevalencia del sexting por edades (tercer objetivo), se observa concordancia entre los resultados encontrados y los estudios que coinciden en señalar que esta práctica se acrecienta con la edad (Capafóns, 2014; Dake et al., 2012; Döring, 2014; Gámez-Guadix et al., 2017; Klettke et al., 2014). Uno de los últimos datos recogidos en España sobre participación en sexting de menores con edades comprendidas entre 11 y 16 años, señala que al menos $31 \%$ reconoce haberlo practicado durante el último año (Jiménez, Garmendia \& Casado, 2018). Comparando con el índice de participación hallado en el presente estudio, comprobamos una diferencia entre edades que supone un incremento del $10.6 \%$ en el índice de participación. Sin embargo, se constata que ese incremento no es continuo, pues se observa una subida progresiva hasta los 20 años, pero a partir de ahí aparece una tendencia decreciente; de manera que el índice de participación a los 24 años es ligeramente inferior al de los 18 años. Sería interesante confirmar si es un cambio meramente circunstancial o podría tener alguna explicación causal como, por ejemplo, la influencia de la propia formación universitaria.

El incremento de la preocupación social por conductas de riesgo entre jóvenes, como en el caso del sexting, hace necesario aproximarse al fenómeno de manera rigurosa para tratar de comprender la forma en que los jóvenes manifiestan su participación en tales prácticas. En España, los estudios sobre sexting son escasos y la mayoría están centrados en la población adolescente, por ello pensamos que la principal aportación del estudio radica en poner de manifiesto las características más significativas de esta práctica en jóvenes universitarios. Dado que es una conducta de riesgo que puede tener consecuencias impredecibles sobre el presente y el futuro, tanto personal como profesional, se hace necesario recomendar prudencia y responsabilidad en su uso; siendo precisamente en la toma de conciencia de sus repercusiones donde deberían incidir los profesionales de la psicología, sobre todo en jóvenes con edades previas a la universitaria.

Entre las limitaciones del estudio conviene subrayar que han participado estudiantes de una sola universidad, lo cual resta representatividad a los resultados; asimismo, su carácter transversal limita las posibilidades de llegar a conclusiones más consistentes, por lo que consideramos recomendable plantear estudios futuros de tipo longitudinal y que incorporen muestras de otras universidades.

\section{Referencias}

Agustina, J. R., \& Gómez-Durán, E. L. (2012). Sexting: Research criteria of a globalized social phenomenon. Archives Sexual Behavior, 41(6), 1325-1328. http://dx.doi.org/10.1007/s10508012-0038-0

Agustina, J. R., \& Gómez-Durán, E. L. (2016). Factores de riesgo asociados al sexting como umbral de diversas formas de victimización. Estudio de factores correlacionados con el sexting en una muestra universitaria. IDP. Revista de Internet, Derecho y Política, 22, 21-47.
Albury, K., Funnell N., \& Noonan, E. (2010), The politics of sexting: Young people, self-representation and citizenship. En McCallum, K. (Ed.), Media, democracy and change: Refereed proceedings of the Australian and New Zealand Communications Association Annual Conference, Canberra, July 7-9. Recuperado de http://www.anzca.net/conferences/past-conferences/94-anzca10proceedings.html.

Alonso, P. (2017). Evaluación del fenómeno del sexting y de los riesgos emergentes de la red en adolescentes de la provincia de Ourense (Tesis doctoral). Universidad de Vigo.

APA. (2017). Ethical principles of psychologists and code of conduct. Washington: American Psychological Association. Recuperado de https://www.apa.org/ethics/code/ethics-code-2017.pdf.

Ballester, R., Gil, M. D., Gómez, S., \& Gil, B. (2010). Propiedades psicométricas de un instrumento de evaluación de la adicción al cibersexo. Psicothema, 22(4), 1048-1053. http://dx.doi. org/10.4321/s1575-18132007000300009

Benotsch, E. G., Snipes, D. J., Martin, A. M., \& Bull, S. S. (2013). Sexting, substance use, and sexual risk behavior in young adults. Journal of Adolescent Health, 52(3), 307-313. http:// dx.doi.org/10.1016/j.jadohealth.2012.06.011

Capafóns, J. (2014). Informe de adicción al móvil y a internet, ciber-acoso, acoso sexual y sexting en adolescentes de Tenerife. Recuperado de http://www.internetsinriesgos.com/wp-content/uploads/2013/10/Anexo-8.-Informe-adiccion-al-movil-y-a-internet-ciberacoso-sexting.pdf.

Cataño, D., Restrepo, S. A., Portilla, N. D., \& Ramírez, H. D. (2008). Autoestima y sexualidad en adolescentes: validación de una escala. Investigación Andina, 10(16), 57-66.

Chacón-López, H., Romero-Barriga, J. F., Aragón-Carretero, Y., \& Caurcel-Cara, M. J. (2016). Construcción y validación de la Escala de Conductas sobre Sexting (ECS). Revista Española de Orientación y Psicopedagogía, 27(2), 99-115. http://dx.doi. org/10.5944/reop.vol.27.num.2.2016.17116

Chalfen, R. (2009). 'It'sonlyapicture': Sexting, 'smutty' snapshotsand felony charges. Visual Studies, 24(3), 258-268. http://dx.doi. org/10.1080/14725860903309203

Dake, J. A., Price, J. H., Maziarz, L., \& Ward, B. (2012). Prevalence and correlates of sexting behavior in adolescents. American Journal of Sexuality Education, 7(1), 1-15. http://dx.doi.org/10 $.1080 / 15546128.2012 .650959$

Deb-Levine, M. A. (2013). Sexting: A terrifying health risk ... or the new normal for young adults? Journal of Adolescent Health, 52, 257-258. http://dx.doi.org/10.1016/j.jadohealth.2013.01.003

Delevi, R., \& Weisskirch, R. S. (2013). Personality factors as predictors of sexting. Computers in Human Behavior, 29(6), 25892594. http://dx.doi.org/10.1016/j.chb.2013.06.003

Dir, A. L., Cokunpinar, A., Steiner, J. L., \& Cyders, M. A. (2013). Understanding differences in sexting behaviors across gender, relationship status, and sexual identity, and the role of expectancies in sexting. Cyberpsychology Behavior and Social Networking, 6(8), 568-574. http://dx.doi.org/10.1089/cyber.2012.0545

Dir, A. L., Cyders, M. A., \& Coskunpinar, A. (2013). From the bar to the bed via mobile phone: A first test of the role of problematic alcohol use, sexting, and impulsivity-related traits in sexual hookups. Computers in Human Behavior, 29(4), 1664-1670. http://dx.doi.org/10.1016/j.chb.2013.01.039

Dobson, A. (2014). Laddishness online: The possible significations and significance of 'performative shamelessness' for young women in the post-feminist context. Cultural Studies, 28(1), 142-164.

Dobson, A. (2015). Postfeminist digital cultures: Femininity, social media, and self-representation. New York: Palgrave Macmillan. 
Döring, N. (2014). Consensual sexting among adolescents: Risk prevention through abstinence education or safer sexting? Cyberpsychology: Journal of Psychosocial Research on Cyberspace, 8(1), article 9. http://dx.doi.org/10.5817/CP2014-1-9

Drouin, M., \& Landgraff, C. (2012). Texting, sexting, and attachment in college students' romantic relationships. Computers in Human Behavior, 28(2), 444-449. http://dx.doi.org/10.1016/j. chb.2011.10.015

Drouin, M., Vogel, K. N., Surbey, A., \& Stills, J. R. (2013). Let's talk about sexting, baby: Computer-mediated sexual behaviors among young adults. Computers in Human Behavior, 29(5), 2530. http://dx.doi.org/10.1016/j.chb.2012.12.030

Durham, M. (2008), The Lolita effect: The media sexualisation of young girls and what we cane do about it. London: Gerald Duchworth Press.

Englander, E. (2012), Low risk associated with most teenage sexting: A study of 617 18-yearolds. Massachusetts Aggression Reduction Center: Bridgewater State University.

Gámez-Guadix, M., Almendros, C., Borrajo, E., \& Calvete, E. (2015). Prevalence and association of sexting and online sexual victimization among Spanish adults. Sexuality Research and Social Policy, 12(2), 145-154. http://dx.doi.org/10.1007/ s13178-015-0186-9

Gámez-Guadix, M., Borrajo, E., \& Almendros, C. (2016). Risky online behaviors among adolescents: Longitudinal relations among problematic Internet use, cyberbullying perpetration, and meeting strangers online. Journal of Behavioral Addictions, 5(1), 100-107. http://dx.doi.org/10.1556/2006.5.2016.013

Gámez-Guadix, M., Santisteban, P., \& Resett, S. (2017). Sexting among Spanish adolescents: Prevalence and personality profiles. Psicothema, 29(1), 29-34. http://dx.doi.org/10.7334/psicothema2016.222

García-Gómez, A. (2017). Teen girls and sexual agency: Exploring the intrapersonal and intergroup dimensions of sexting. Media, Culture and Society, 39(3), 391-407.

García-Gómez, A. (2018). From selfies to sexting: Tween girls, intimacy, and subjectivities. Girlhood Studies, 11(1), 43-58. Special issue: Locating Tween girls. Edited by Melanie Kennedy and Natalie Coulter.

García-Gómez, A. (2019). Sexting and hegemonic masculinity: Interrogating male sexual agency, empowerment and dominant gendered norms. En Garcés-Conejos Blitvich, P., \& Bou-Franch, P. (Eds.), Analyzing digital discourse: New insights and future directions (pp. 313-339). London: Palgrave MacMillan.

Gill, R. (2012). Media, empowerment and the "sexualisation of culture" debates. Sex Roles. A Journal of Research, 66(11-12), 736-745.

Gordon-Messer, D., Bauermeister, J. A., Grodzinski, A., \& Zimmerman, M. (2013). Sexting among young adults. Journal of Adolescent Health, 52(3), 301-306. http://dx.doi.org/10.1016/j. jadohealth.2012.05.013

Hasinoff, A. A. (2012). Sexting as media production: Rethinking social media and sexuality. New Media \& Society, 15(4), 449-465.

Hasinoff, A. A. (2014). Blaming sexualization for sexting. Girlhood Studies, 7(1), 102-120.

Hasinoff, A. A. (2015). Sexting panic: Rethinking criminalization, privacy, and consent. Champaign: University of Illinois Press.

Hudson, H. K. (2011). Factors affecting sexting behaviours among selected undergraduate students. (Tesis doctoral no publicada), Southern University Illinois Carbondale, Illinois, Estados Unidos.

Inteco. (2011). Guía sobre adolescencia y sexting: qué es y cómo prevenirlo. Madrid: Instituto Nacional de Tecnologías de la Comunicación. Recuperado de http://www.educacion.navarra.
es/portal/digitalAssets/49/49142_20110337.pdf.

Jiménez, E., Garmendia, M., \& Casado, M. A. (2018). Entre selfies y whatsapps. Barcelona: Editorial Gedisa.

Klettke, B., Hallford, D. J., \& Mellor, D. J. (2014). Sexting prevalence and correlates: A systematic literature review. Clinical Psychology Review, 34(1), 44-53. http://dx.doi.org/10.1016/j. cpr.2013.10.007

Lee, M., \& Crofts, T. (2015). Gender, pressure, coercion and pleasure: Untangling motivations for sexting between young people. British Journal of Criminology, 55(3), 454-473.

Livingstone, S. (2008). Taking risky opportunities in youthful content creation: Teenagers' use of social networking sites for intimacy, privacy and self-expression. New Media \& Society, 10(3), 393-411.

Marcum, C. D., Higgins, G. E., \& Ricketts, M. L. (2014). Sexting behaviors among adolescents in rural North Carolina: A theoretical examination of low selfcontrol and deviant peer association. International Journal of Cyber Criminology, 8(2), 68-78.

Mitchell, K. J., Finkelhor, D., Jones, L. M., \& Wolak, J. (2012). Prevalence and characteristics of youth sexting: A national study. Pediatrics, 129(1), 13-20. http://dx.doi.org/10.1542/peds.20111730

Morelli, M., Bianchi, D., Baiocco, R., Pezzuti, L., \& Chirumbolo, A. (2016). Sexting, psychological distress and dating violence among adolescents and young adults. Psicothema, 28(2), 137142. http://dx.doi.org/10.7334/psicothema2015.193

O’Neal, P., Cummings, T., Hardy, C., \& Ott, M. A. (2013). Predictors of sexting in a university population. Journal of Adolescents Health, 52(2), Suplemento 1, 87. http://dx.doi.org/10.1016/j. jadohealth.2012.10.204

Perkins, A. B., Becker, J. V., Tehee, M., \& Mackelprang, E. (2014). Sexting behaviors among college students: Cause for concern? International Journal of Sexual Health, 26(2), 79-92. http:// dx.doi.org/10.1080/19317611.2013.841792

Quesada, S., Fernández-González, L., \& Calvete, E. (2018). El sexteo (sexting) en la adolescencia: frecuencia y asociación con la victimización de ciberacoso y violencia en el noviazgo. Behavioral Psychology/Psicología Conductual, 26(2), 225-242.

Reyns, B. W., Henson, B., \& Fisher, B. S. (2014). Digital deviance: Low self-control and opportunity as explanations of sexting among college students. Sociological Spectrum: Mid-South Sociological Association, 34(3), 273-292. http://dx.doi.org/10.108 $0 / 02732173.2014 .895642$

Ringrose, J., Harvey, L., Gill, R., \& Livingstone, S. (2013). Teen girls, sexual double standards and 'sexting': Gendered value in digital image exchange. Feminist Teory, 14(3), 305-323. http:// doi.org/10.1177/1464700113499853

Rosenberg, M. (1965). Society and the adolescent self-image. Princeton: Princeton University Press.

Stasko, E. (2018). Sexting ethics in young people's digital cultures: Risk, shame and the negotiation of privacy and consent (Tesis doctoral), Universidad de Surrey.

Vázquez-Morejón, A. J., Jiménez García-Bóveda, R., \& Vázquez-Morejón Jiménez, R. (2004). Escala de Autoestima de Rosenberg: fiabilidad y validez en población española. Apuntes de Psicología, 22(2), 247-255.

Ybarra, M. L., \& Mitchell, K. J. (2014). "Sexting" and its relation to sexual activity and sexual risk behavior in a national survey of adolescents. Journal of Adolescent Health, 55(6), 757-764. http://doi.org/10.1016/j.jadohealth.2014.07.012 